\title{
Automatic evaluation of myocardial perfusion on SPECT: Need for "Normality"
}

\author{
Riccardo Liga, $M D{ }^{a}$ and Alessia Gimelli, $M D^{b}$ \\ a University of Pisa, Pisa, Italy \\ b Fondazione Toscana/CNR G. Monasterio, Pisa, Italy
}

Received Oct 5, 2017; accepted Oct 5, 2017

doi:10.1007/s12350-017-1097-3

\section{See related article, pp. 775-785}

Myocardial perfusion imaging (MPI) with singlephoton emission computer tomography (SPECT) is still the most performed and, probably, the most trusted noninvasive imaging modality for the evaluation of patients with suspected or known ischemic heart disease (IHD). Despite the great advancement of all the other imaging modalities that has taken place in the last two decades, the ability of SPECT MPI in terms of in-depth characterization and risk stratification of patients with IHD remains unbeaten, with solid evidence proving its additive value in a wide range of patients subsets (i.e., symptomatic vs asymptomatic subjects; normal weight vs obese; sinus vs paced rhythm $)^{1-4}$ and clinical scenarios (i.e., chronic IHD vs acute coronary syndromes). 5,6 In particular, among the major advantages of this technology, the intrinsic versatility of SPECT MPI is probably the most relevant. In fact, it can be easily coupled with any of the currently available stress tests (i.e., exercise, vasodilators, dobutamine), allowing obtaining reproducible measures of myocardial ischemic susceptibility in practically every category of patients. ${ }^{1,7}$ Moreover, SPECT MPI study can provide information on LV structure and function that may both contribute to the diagnostic process, and provide independent measures of adverse patients prognosis. ${ }^{8,9}$

Nevertheless, even with the evident advantages of nuclear cardiac imaging, SPECT MPI is also characterized by some relevant drawbacks that should be

Reprint requests: Alessia Gimelli, MD, Fondazione Toscana/CNR G. Monasterio, Pisa, Italy; gimelli@ftgm.it

J Nucl Cardiol 2019;26:786-9.

1071-3581/\$34.00

Copyright (C) 2017 American Society of Nuclear Cardiology. always taken into account, particularly if traditional Anger cameras are employed. Above all, the relatively high radiation burden of a stress/rest imaging protocol performed with a classical SPECT camera equipped with $\mathrm{Na} / \mathrm{I}$ scintillating crystals-especially if ${ }^{201} \mathrm{Tl}$ was used as radiotracer instead of the more favorable ${ }^{99 \mathrm{~m}} \mathrm{Tc}$ based compounds-has always represented a theoretical limitation of this imaging modality, ${ }^{10}$ making the clinician often favor other non-ionizing non-invasive myocardial imaging techniques. Secondly, the relatively lengthy acquisition protocol of traditional myocardial SPECT imaging, still not too infrequently performed on a two-day basis, has made the other non-invasive imaging techniques look more appealing. ${ }^{1,10}$ In the last decade, both those limitations have been practically solved by several improvements consistently both the architecture and software settings of traditional Anger cameras. $^{11}$

Regarding innovation in technology, a substantial increase of photon sensitivity and spatial resolution can be also obtained by the implementation of confocal collimators mounted on conventional dual-headed gamma cameras (i.e., IQ SPECT). ${ }^{12}$ These collimators are characterized by an intrinsically more convergent field of view at the center, while the convergence is reduced approaching the edges of the field of view. The increased sensitivity of those devices allows reducing the acquisition times to about 4 minutes per scan, with a similar quality of traditional systems. ${ }^{12,13}$

Finally, the introduction of dedicated cardiac cameras built around solid-state Cadmium-Zinc-Telluride (CZT) detectors has represented a real revolution in the field of nuclear cardiac imaging, allowing reducing consistently both the radiation burden and acquisition time of MPI, while maintaining if not improving the overall diagnostic capability. ${ }^{7,14,15}$

Because of the absence of the photomultipliers tubes, CZT cameras are consistently less bulky than traditional SPECT devices, increasing patients comfort 
while reducing overall logistic needs. Nevertheless, while performing consistently better than Anger cameras, CZT devices still constitute a limited fraction of the working nuclear cardiac cameras, mainly because of the higher initial costs of this technology.

On the other hand, nuclear cardiac imaging on conventional SPECT devices has been also interested by a consistent software evolution, mainly based on iterative reconstruction algorithms that outperform the "'old" filtered back projection (FBP) in terms of higher image quality, with less background noise, and smoother myocardial borders. ${ }^{16}$ Image quality is further improved by the implementation of resolution recovery algorithms, additively reducing image noise with respect to conventional reconstruction software. While the additive value of novel iterative resolution recovery (IRR) reconstruction algorithm may be limited in high-count SPECT studies, it becomes relevant in the case of lowdose SPECT scans, where the improvement with respect to FBP becomes more relevant. ${ }^{17}$ In fact, low-dose cardiac images acquired with IRR software are superior to those acquired with FBP, allowing reducing acquisition times and/or injected radiotracers dose. It is not surprising that in the last years the feasibility of lowdose SPECT imaging protocols with traditional Anger cameras has been demonstrated, allowing maintaining excellent image quality and diagnostic accuracy while reducing consistently the overall radiation burden of the scan. ${ }^{18}$ However, the technical performance of conventional SPECT devices in the new realm of low-dose scans has to be still characterized, since the application the old rules that were appropriate in the case of fulldose acquisitions might not be appropriate in this novel setting.

In this number of the Journal, Scabbio et al. evaluate the possible clinical impact of the implementation of low-dose SPECT studies on the automatic quantification of myocardial perfusion information. ${ }^{19}$ The main objective of the study was the analysis of the impact of non-study-specific software database-derived from a reference population imaged with a different acquisition protocol and injected dose-on the accurate quantification of regional myocardial perfusion abnormalities in patients imaged with a traditional dual-headed SPECT camera that used a dedicated reconstruction algorithm with IRR technology.

In this setting, the introduction of reliable quantitative software for the automatic evaluation of myocardial perfusion heterogeneity has been a great advancement in modern nuclear cardiology, with different algorithms currently available from a number of vendors. ${ }^{20}$ While the different software differ in terms of the specific algorithm used for the quantification process, the basic steps of $\mathrm{LV}$ perfusion analysis are the $3 \mathrm{D}$ segmentation of the myocardium, the generation of polar maps of relative myocardial count density, and the comparison of the obtained polar map with average values obtained in a reference population. Unfortunately, while site- or protocol-specific normal limits should be created using generally a number of SPECT scans of patients with a low $(<5 \%)$ pre-test likelihood of CAD, most of the times the original vendor-provided normality databases are employed, possibly introducing relevant bias in the evaluation of myocardial perfusion parameters.

However, to date, the impact of the use of nonspecific normality database for the analysis of cardiac SPECT with novel IRR reconstruction algorithms and dose-sparing protocols had not been evaluated.

In order to accomplish this task, the study by Scabbio et al. includes both a pre-clinical assessment with an anthropometric phantom and a clinical validation on a real-life population of patients submitted to clinically indicated stress/rest myocardial SPECT studies.

In the phantom study, the impact of different radiotracer dose regimens and of specific reconstruction algorithms (FBP, OSEM, and IRR) on automatic myocardial perfusion analysis was evaluated, showing that even significant reduction of the injected dose (i.e., $25 \%$ of the traditional radiotracer dose) did not translate into significant errors in the regional evaluation of myocardial perfusion on polar maps, with only minor regional variations when IRR algorithms were applied. For the patient-based study, 40 consecutive normal weight patients and an identical number of overweight subjects were selected, and the perfusion scans were analyzed with either IRR and non-IRR reconstruction software using different normality databases (i.e., full counts and half counts). The main results of the study were that the selection of the specific normality database of myocardial perfusion had a significant impact on the quantification of regional myocardial perfusion heterogeneity, both at rest and after stress. This finding was more evident in the case of low-dose studies as well as in the overweight subjects, with a significantly adverse impact on the accurate evaluation of stress-induced myocardial perfusion abnormalities. Moreover, significant difference in the outputs obtained with IRR and non-IRR reconstruction software was reported, confirming the protocol-specificity of automatic SPECT perfusion analysis. Interestingly, the impact of dose reduction on the accuracy of myocardial perfusion analysis was particularly evident only in the case of " "extremely" low-dose protocols ( $25 \%$ of the traditional injected dose) and in overweight patients, while it remained non-significant when normal weight subjects were imaged. In the former setting, even the use of a 
half-counts normality database for automatic perfusion analysis could not eliminate the bias in the quantification of myocardial perfusion heterogeneity.

The authors of the study should be commended for having conducted a technically solid study that addressed a topic of high clinical interest.

The peculiar analysis that was performed-the use of three different image reconstructions with progressively reduced acquisition times for SPECT projection-allowed obtaining for the same population of patients three sets of images that could mimic accurately normal dose, half dose, and quarter dose scans. ${ }^{21}$ Accordingly, the obtained results indicated that the radical reduction of the injected radiotracer dose, as currently feasible with high-end SPECT systems, must be also paralleled by an improvement of software settings, particularly if automatic perfusions analysis is attempted.

In this respect, automatic quantification software offer the chance to obtain accurate and highly reproducible assessment of myocardial perfusion heterogeneity that compete well, and generally outperform, the performance of visual segmental scoring, even when performed by expert readers. ${ }^{20}$ These algorithms ideally offer the chance to unmask subtle changes of regional LV ischemic burden on sequential myocardial perfusion scans, as a frequent condition in many cardiac patients, potentially guiding targeted therapeutic interventions. However, while automatic quantitative perfusion analysis is the rule in conditions where an absolute evaluation of frequently minimal changes of myocardial perfusion heterogeneity is mandatoryrandomized controlled trial settings with sequential SPECT scans (i.e., before and after a therapeutic intervention) ${ }^{22,23}$ - they are less frequently employed in daily clinical practice, where visual scoring of myocardial perfusion is generally performed. Nevertheless, comparative analyses have shown how automatic perfusion analysis of SPECT scans may be more accurate than visual scoring in diagnosing the presence of significant CAD, obviating the need of frequently cumbersome image reconstructions. $^{20}$

Beside the many advantages, the wide implementation of automatic quantification perfusion software is limited by some relevant pitfalls of these technology, namely the need of a reference database of normal patients scanned with the same imaging protocol and the great susceptibility from image artifacts. As a matter of fact, a significant difference in terms of protocol characteristics between the normality database and the study patients may impact profoundly the outputs of automatic perfusion analysis, and, as demonstrated by Scabbio et al., may be more relevant in overweight patients or when IRR algorithms are employed. ${ }^{18,24}$ On the other hand, the presence of any image artifact, while generally ignored by expert visual readers, may be misdiagnosed by automatic quantification software, especially when attenuation correction is not available.

While adding some interesting information on these topics, the study by Scabbio et al. has also some limitations that prevent the wide generalizability of its findings. First of all, the occurrence of attenuation artifacts not corrected by any form of attenuation correction algorithms might have diminished the reproducibility of automatic perfusion analysis, reducing the comparability of the different acquisition protocols of SPECT images. ${ }^{20}$ Second, the absence of any clinically meaningful reference standard for the evaluation of myocardial perfusion analysis (i.e., coronary angiography) does not allow deriving any definitive conclusion on the results, whose clinical impact is still not completely defined. In conclusion, present and previous reports further underline the great advancements that nuclear cardiac imaging has had in the last decade, bringing this technique into an era characterized by reduced patient dosimetry and ultra-fast acquisition protocols. However, further improvements in software settings are needed to allow the full exploitation of the diagnostic potential of myocardial perfusion imaging. Above all, the need of different reference perfusion databases of normal patients studied with variable protocols and under various study conditions has been strongly remarked in order to allow a proper definition of subtle alteration of myocardial perfusion heterogeneity and definition of patient risk also in patients studied with low-dose protocols and/or with innovative IRR reconstruction algorithms.

\section{Conflict of Interest}

The authors declare that no potential conflict of interest exists and that all them have read and approved the manuscript.

\section{Disclosure}

The authors have nothing to declare.

\section{References}

1. Underwood SR, Anagnostopoulos C, Cerqueira M, Ell PJ, Flint EJ, Harbinson M, et al. Myocardial perfusion scintigraphy: The evidence. Eur J Nucl Med Mol Imaging 2004;31:261-91.

2. Acampa W, Gaemperli O, Gimelli A, Knaapen P, Schindler TH, Verberne $\mathrm{H}$, et al. Role of risk stratification by SPECT, PET, and hybrid imaging in guiding management of stable patients with ischaemic heart disease: Expert panel of the EANM cardiovascular committee and EACVI. Eur Heart $\mathrm{J}$ Cardiovasc Imaging 2015;16:1289-98. 
3. Gimelli A, Bottai M, Giorgetti A, Genovesi D, Filidei E, Marzullo P. Evaluation of ischaemia in obese patients: Feasibility and accuracy of a low-dose protocol with a cadmium-zinc telluride camera. Eur J Nucl Med Mol Imaging 2012;39:1254-61.

4. Lapeyre AC 3rd, Poornima IG, Miller TD, Hodge DO, Christian TF, Gibbons RJ. The prognostic value of pharmacologic stress myocardial perfusion imaging in patients with permanent pacemakers. J Nucl Cardiol 2005;12:37-42.

5. Douglas PS, Hoffmann U, Patel MR, Mark DB, Al-Khalidi HR, Cavanaugh B, et al. Outcomes of anatomical versus functional testing for coronary artery disease. N Engl J Med 2015;372:1291-300.

6. Nabi F, Kassi M, Muhyieddeen K, Chang SM, Xu J, Peterson LE, et al. Optimizing evaluation of patients with low-to-intermediaterisk acute chest pain: A randomized study comparing stress myocardial perfusion tomography incorporating stress-only imaging versus cardiac CT. J Nucl Med 2016;57:378-84.

7. Gimelli A, Liga R, Pasanisi EM, Casagranda M, Coceani M, Marzullo P. Influence of cardiac stress protocol on myocardial perfusion imaging accuracy: The role of exercise level on the evaluation of ischemic burden. J Nucl Cardiol 2016;23:1114-22.

8. Gimelli A, Liga R, Giorgetti A, Kusch A, Pasanisi EM, Marzullo P. Relationships between myocardial perfusion abnormalities and poststress left ventricular functional impairment on cadmium-zinctelluride imaging. Eur J Nucl Med Mol Imaging 2015;42:9941003.

9. Gimelli A, Liga R, Clemente A, Marras G, Kusch A, Marzullo P. Left ventricular eccentricity index measured with SPECT myocardial perfusion imaging: An additional parameter of adverse cardiac remodeling. J Nucl Cardiol 2017. doi:10.1007/s12350-017-0777-3.

10. Einstein AJ, Pascual TN, Mercuri M, Karthikeyan G, Vitola JV, Mahmarian JJ, et al. Current worldwide nuclear cardiology practices and radiation exposure: Results from the 65 country IAEA nuclear cardiology protocols cross-sectional study (INCAPS). Eur Heart J 2015;36:1689-96.

11. Slomka PJ, Dey D, Duvall WL, Henzlova MJ, Berman DS, Germano G. Advances in nuclear cardiac instrumentation with a view towards reduced radiation exposure. Curr Cardiol Rep 2012;14:208-16.

12. Gremillet E, Agostini D. How to use cardiac IQ•SPECT routinely? An overview of tips and tricks from practical experience to the literature. Eur J Nucl Med Mol Imaging 2016;43:707-10.

13. Caobelli F, Ren Kaiser S, Thackeray JT, Bengel FM, Chieregato $\mathrm{M}$, Soffientini A, et al. IQ SPECT allows a significant reduction in administered dose and acquisition time for myocardial perfusion imaging: Evidence from a phantom study. J Nucl Med 2014;55:2064-70.
14. Nakazato R, Berman DS, Hayes SW, Fish M, Padgett R, Xu Y, et al. Myocardial perfusion imaging with a solid-state camera: Simulation of a very low dose imaging protocol. J Nucl Med 2013;54:373-9.

15. Nkoulou R, Fuchs TA, Pazhenkottil AP, Kuest SM, Ghadri JR, Stehli J, et al. Absolute myocardial blood flow and flow reserve assessed by gated SPECT with cadmium-zinc-telluride detectors using ${ }^{99 \mathrm{~m}}$ Tc-tetrofosmin: Head-to-head comparison with $13 \mathrm{~N}$ ammonia PET. J Nucl Med 2016;57:1887-92.

16. Zoccarato O, Scabbio C, De Ponti E, Matheoud R, Leva L, Morzenti S, et al. Comparative analysis of iterative reconstruction algorithms with resolution recovery for cardiac SPECT studies. A multi-center phantom study. J Nucl Cardiol 2014;21:135-48.

17. DePuey EG. Advances in SPECT camera software and hardware: Currently available and new on the horizon. J Nucl Cardiol 2012;19:551-81.

18. Marcassa C, Campini R, Zoccarato O, Calza P. Wide beam reconstruction for half-dose or half-time cardiac gated SPECT acquisitions: Optimization of resources and reduction in radiation exposure. Eur J Nucl Med Mol Imaging 2011;38:499-508.

19. Scabbio C, Zoccarato O, Malaspina S, Lucignani G, Del Solec A, Lecchia M. Impact of non-specific normal databases on perfusion quantification of lowdose myocardial SPECT studies. J Nucl Cardiol 2017. doi:10.1007/s12350-017-1079-5.

20. Slomka P, Xu Y. Berman D and Germano G Quantitative analysis of perfusion studies: Strengths and pitfalls. J Nucl Cardiol 2012;19:338-46.

21. Lecchi M, Martinelli I, Zoccarato O, Maioli C, Lucignani G, Del Sole A. Comparative analysis of full-time, half-time, and quartertime myocardial ECG-gated SPECT quantification in normalweight and overweight patients. J Nucl Cardiol 2017;24:876-87.

22. Shaw LJ, Berman DS, Maron DJ, Mancini GB, Hayes SW, Hartigan PM, et al. Optimal medical therapy with or without percutaneous coronary intervention to reduce ischemic burden: Results from the Clinical Outcomes Utilizing Revascularization and Aggressive Drug Evaluation (COURAGE) Trial Nuclear Substudy. Circulation 2008;117:1283.

23. Mahmarian JJ, Cerqueira MD, Iskandrian AE, Bateman TM, Thomas GS, Hendel RC, et al. Regadenoson induces comparable left ventricular perfusion defects as adenosine: A quantitative analysis from the ADVANCE MPI 2 trial. JACC Cardiovasc Imaging 2009;2:959-68.

24. Zoccarato O, Marcassa C, Lizio D, Leva L, Lucignani G, Savi A, et al. Differences in polar-map patterns using the novel technologies for myocardial perfusion imaging. J Nucl Cardiol 2016. doi:10.1007/s12350-016-0500-9. 